\title{
Robust expansion of phylogeny for fast-growing genome sequence data
}

\section{Authors}

5 Yongtao $\mathrm{Ye}^{1,2^{*}}$, Marcus H. Shum ${ }^{1,2^{*}}$, Joseph L. Tsui ${ }^{1,2^{*}}$, Guangchuang Yu ${ }^{3 *}$, David K. Smith ${ }^{1}$,

6 Huachen Zhu ${ }^{1,2,4,5}$, Joseph T. Wu ${ }^{1,2}$, Yi Guan ${ }^{1,2,4,5}$, Tommy T. Lam ${ }^{1,2,4,5,6}$

8 Affiliations

$10{ }^{1}$ State Key Laboratory of Emerging Infectious Diseases, School of Public Health, The University

11 of Hong Kong, Hong Kong SAR, P. R. China

$12{ }^{2}$ Laboratory of Data Discovery for Health Limited, 19W Hong Kong Science \& Technology

13 Parks, Hong Kong SAR, P. R. China

$14{ }^{3}$ Department of Bioinformatics, School of Basic Medical Sciences, Southern Medical University, 15 Guangzhou, Guangdong, China

$16{ }^{4}$ Guangdong-Hongkong Joint Laboratory of Emerging Infectious Diseases, Joint Institute of 17 Virology (Shantou University/The University of Hong Kong), Shantou, Guangdong, 515063, P. 18 R. China.

$19{ }^{5}$ EKIH (Gewuzhikang) Pathogen Research Institute, Futian District, Shenzhen City, Guangdong, 20 518045, P. R. China.

$21{ }^{6}$ Centre for Immunology \& Infection Limited, 17W Hong Kong Science \& Technology Parks, 22 Hong Kong SAR, P. R. China

$24 *$ These authors contributed equally to the research.

25 \# Correspondence: ttylam@hku.hk 


\section{Abstract}

28 Massive sequencing of SARS-CoV-2 genomes has led to a great demand for adding new samples

29 to a reference phylogeny instead of building the tree from scratch. To address such challenge, we

30 proposed an algorithm 'TIPars' by integrating parsimony analysis with pre-computed ancestral

31 sequences. Compared to four state-of-the-art methods on four benchmark datasets (SARS-CoV-2,

32 Influenza virus, Newcastle disease virus and 16S rRNA genes), TIPars achieved the best

33 performance in most tests. It took only 21 seconds to insert 100 SARS-CoV-2 genomes to a 100k-

34 taxa reference tree using near 1.4 gigabytes of memory. Its efficient and accurate phylogenetic

35 placements and incrementation for phylogenies with highly similar and divergent sequences

36 suggest that it will be useful in a wide range of studies including pathogen molecular

37 epidemiology, microbiome diversity and systematics. 
41 Next generation sequencing (NGS) technologies enable large-scale exploration of diversity and

42 monitoring temporal evolution of organisms, which often involve generating and analyzing large

43 numbers of sequences from new organisms on an ongoing basis. For instance, over 5 million of

44 SARS-CoV-2 genomes have been sequenced within two years of the pandemic (Shu \&

45 McCauley, 2017), largely facilitating transmission tracking and disease control. Conventional

46 methods of phylogeny inference from scratch such as those implemented in IQ-TREE2 (Minh et

47 al., 2020) and FastTree2 (Price, Dehal, \& Arkin, 2010) could hardly cope with such rapidly

48 growing huge sequence datasets. Therefore, determining the evolutionary position of new

49 sequences as they become available by placing or inserting them into the reference tree becomes a

50 more efficient alternative. Such 'phylogenetic placement' has been useful for taxonomic

51 classification, while accumulative addition of sequences (incrementing the phylogeny as a result)

52 allow efficient update of the growing phylogeny in a global context.

54 Previously published methods such as PhyClass (Filipski, Tamura, Billing-Ross, Murillo, \&

55 Kumar, 2015), EPA-ng (Barbera et al., 2019) and pplacer (Matsen, Kodner, \& Armbrust, 2010)

56 utilize minimum evolution or maximum likelihood criteria to infer the evolutionary position of

57 the query sequence and place it directly onto a pre-built phylogeny. These algorithms require

58 relatively large computer memory or long runtime which makes massive sequence insertion

59 difficult. Recently, in respect of tracking the diversity of the large amount of SARS-CoV-2 virus

60 genomes, UShER (Yatish Turakhia et al., 2021) was developed to tackle this problem by

61 calculating the 'branch parsimony score' to search for positions of taxa placement only based on

62 sequence mutations to a particular reference. It is extremely fast as compared to the other existing

63 programs. Although the performance of UShER on the SARS-CoV-2 genomes is promising, the 
64 placement performance for genome sequences with greater divergence is not well studied.

66 We hereby introduce a new approach TIPars, which inserts sequences into a reference phylogeny

67 based on parsimony criterion with the aids of a full multiple sequence alignment of taxa and pre-

68 computed ancestral sequences. The ancestral sequences are useful and efficient in assisting the

69 search of the best placed position because these ancestral sequences often contain rich

70 information in the evolution context of a phylogenetic tree (Loytynoja, Vilella, \& Goldman,

71 2012). Recent ancestral sequence reconstruction methods such as PastML (Ishikawa, Zhukova,

72 Iwasaki, \& Gascuel, 2019) and RASP4 (Y. Yu, Blair, \& He, 2020) have improved speed and

73 accuracy to become feasible in the huge SARS-CoV-2 phylogeny. TIPars searches the position

74 for insertion by calculating the triplet-based minimal substitution score for the query sequence on

75 all branches (Fig. 1A). To compare the performances of different phylogenetic

76 placement/insertion methods including TIPars, UShER, EPA-ng, IQ-TREE2 and PAGAN2

77 (Loytynoja et al., 2012), we applied them on four benchmark datasets (SARS-CoV-2, Influenza

78 virus, Newcastle disease virus and 16S rRNA genes). The first test is single taxon placement. We

79 pruned one taxon from a given phylogenetic tree and applied the methods to place it back. The

80 second is multiple taxa insertion in which a set of taxa was removed and sequentially inserted

81 back. We compared the topology and log likelihood for the trees before pruning and after

82 reinsertion. Our evaluation tests aimed to assess the robustness of the methods on both highly

83 similar sequences and divergent sequences, and whether the phylogenetic tree could be efficiently

$84 \quad$ updated with new sequences that are continuously generated.

85

Results 
89 A number of approaches have been proposed for phylogenetic placement or insertion, but dealing

90 with the vast number of SARS-CoV-2 genome sequences has rendered most of these methods

91 impractical or computationally prohibitive. Based on a reference SARS-CoV-2 phylogenetic tree

92 (SARS2-100k) generated from 96,020 unmasked SARS-CoV-2 sequences of high quality (details

93 in Methodology), we evaluated our proposed program TIPars with UShER, EPA-ng, IQ-TREE2

94 and PAGAN2 by sequentially inserting 100 new sequence samples. Only TIPars and UShER

95 were practicable in terms of running time and memory usage. PAGAN2 were not able to

96 complete the insertion within 96 hours and hence no data was available. Although IQ-TREE2

97 used a lower peak memory than EPA-ng, the running time was the highest among all programs. In

98 contrast, EPA-ng achieved a faster running time than IQ-TREE2 but the peak memory usage was

99 around 1 terabyte (TB) which would not be practicable for general users. As for TIPars, it took

100 only 21 seconds (excluding the input loading time) on a 64-cores server and required about 1.4

101 gigabytes (GB) peak memory usage (Table 1). Another computational performance comparison

102 on smaller dataset with 800 bacterial $16 \mathrm{~S}$ rRNA sequences (16S) can be checked in table S1 in

103 which PAGAN2 was runnable. Overall, in the SARS2-100k phylogenetic tree, TIPars ran 10-300

104 folds faster than EPA-ng and PAGAN2 with $98.5 \%$ to $99.9 \%$ less memory used, an efficiency

105 that is comparable to that of the leading program UShER.

\section{Single taxon placement}

Adding a single sequence sample (query) into a reference tree is useful to obtain the phylogenetic placement of the new data, and can be the basic step for expanding the phylogeny with new

111 sequences. We tested TIPars, UShER and EPA-ng on four datasets, including the SARS-CoV-2

112 genomes (SARS2-100k), 16S ribosomal RNA genes (16S), hemagglutinin genes of human 
114 average pairwise genetic distances (substitutions per site) of SARS2-100k and H3N2 are less than

1150.04 (similar sequences) while those of $16 \mathrm{~S}$ and NDV are greater than 0.12 (divergent sequences)

116 (details in Methodology; table S2). For the SARS2-100k dataset, EPA-ng was not applied due to

117 impractically large memory requirement and long runtime.

119 Based on the postorder traversal, between every 10 taxa we selected one sequence from the

120 SARS2-100k sequence alignment resulting in 9,602 sequences, i.e., $10 \%$ of the total taxa in the

121 tree. These selected sequences were individually removed from the reference tree and multiple

122 sequence alignment (MSA) one at a time and used as the query sample for single taxon

123 placement. In datasets of $16 \mathrm{~S}, \mathrm{H} 3 \mathrm{~N} 2$ and NDV, all taxa were removed individually and used for

124 the placement test.

To evaluate the accuracy of each single taxon placement, we calculated the Robinson-Foulds (RF)

distance (Robinson \& Foulds, 1981) between the reference tree before the taxon removal and the

resulting tree after the placement using corresponding programs. An RF distance measures the

topological clustering difference between two trees. A zero RF distance indicates that the two

130 trees are topologically identical, and hence the single taxon placement position is exactly the same

131 as the original position, i.e. a true positive.

133 With the aid of ancestral information and MSA of full sequences, TIPars performed accurately on

134 phylogenies made of highly similar (SARS2-100k and H3N2) and divergent (16S and NDV)

135 sequences (Fig. 1B). However, a drop in accuracy on more divergent sequences was observed

136 from UShER, perhaps because UShER was only based on the sequence mutations to a particular

137 reference sequence as input, which may lose the insertion information (Yatish Turakhia et al.,

138 2021). In addition, we noted that due to the massive sequencing of SARS-CoV-2 by different 
research groups, sequencing quality varies and ambiguity bases often occur in the consensus

genome sequence data, which could affect the placement accuracy. To account for ambiguity data

141 in sequencing, we used a specific substitution scoring table based on the IUPAC nucleotide

142 ambiguity codes (table S3) for the taxon placement and insertion process (details in

143 Methodology), which achieved a robust performance in sequences of different qualities.

145 Notably, when searching through the whole phylogeny for the best position to place a taxon, there may be cases where multiple branches achieve equal minimum substitution scores, and thus the placement will be uncertain. As demonstrated in Fig. 1C, TIPars produced the least number of 148 multiple ambiguously optimal placements in all testing datasets. For example, TIPars generated $23 \%$ fewer multiple placements than UShER in the SARS2-100k dataset.

A possible reason for the relatively poor performance of EPA-ng could be that RF distance may

not be a reliable metric to compare binary trees derived from the phylogeny with polytomy

because there is a very skewed distribution of RF distance when comparing two random binary

trees (Bryant \& Steel, 2009; Lin, Rajan, \& Moret, 2012; Moon \& Eulenstein, 2019). It is notable

that EPA-ng only processes binary trees. To address this issue, a relaxed criterion for true positive

was applied based on whether there are common sister taxa for the removed and re-placed single

taxon, as previously used (Yatish Turakhia et al., 2021). With the adjusted true positive

measurement, TIPars achieved the highest accuracy in all datasets (fig. S1). While the accuracy of

EPA-ng was substantially improved, it was still the lowest among the three tested programs.

161 To assess the practicability for extremely large phylogenies, we applied TIPars and UShER in 162 single taxon placement test over the global SARS-CoV-2 phylogenetic tree with 659,885 masked 
164 Influenza Database (GISAID) (Shu \& McCauley, 2017) on the 6th September 2021. A total of

16565,989 sequences ( $10 \%$ of the total taxa in the tree) were removed and re-inserted individually.

166 Cumulative proportion of single taxon placement result with different RF distance cutoff was

167 shown in Fig. 1D. TIPars produced trees with significantly higher topological similarity to the 168 reference tree with a median RF distance of 0.5 and mean of $5.8(99 \%$ confidence interval $(\mathrm{CI})=$

169 [5.5-6.1]) as compared to UShER (median RF distance is 3.0 and mean is $31.2(99 \% \mathrm{CI}=[30.0-$

$17032.4]))$ at $99 \%$ significance level $\left(\mathrm{p}\right.$-value $\left.<10^{-10}\right)$.

\section{Multiple taxa insertion}

174 Multiple taxa insertion was an alternative method in determining the phylogenetic position of new

175 sequences over conventional complete phylogeny construction from scratch. TIPars and other three programs (IQ-TREE2, PAGAN2 and UShER) were applied on the four datasets to conduct a comprehensive evaluation of performance.

In the SARS2-100k dataset, we performed multiple taxa insertion for 100 sets of $10^{2}$ and $10^{3}$

180 randomly selected sequences (an example is shown in Fig. 2A) (random100 and random1000)

181 and 100 sets of $10^{2}$ and $10^{3}$ successively selected sequences (i.e., a set of successive taxa

182 following the tree postorder traversal; an example is shown in Fig. 2B) (successive100 and 183 successive1000). In the 16S, H3N2 and NDV datasets, 100 sets of 50 sequences were randomly 184 selected. The selected sequences are pruned from the corresponding reference tree and become 185 multiple taxa to be reinserted for each testing set.

187 RF distance and tree log-likelihood (LL) were used to evaluate the performance of the multiple 188 sequence insertion. To evaluate the topology accuracy, the resulting tree produced by the four 
189 programs were compared to the original reference tree (leaf taxa unpruned) to obtain the RF

190 distance. At the same time, Gamma20 log-likelihoods of the reference tree and the resulting tree

191 after optimizing the branch length were also computed using FastTree2 (double-precision version)

192 and their differences were used for evaluation.

194 For the random100 and random1000 datasets, only analyses using TIPars and UShER were able

195 to complete within a reasonable computation time, hence no result from IQ-TREE2 and PAGAN2

196 was present. The resulting trees from multiple taxa insertion using TIPars had a significantly

197 smaller RF distance than those generated using UShER (Fig. 3A). In addition, the log-likelihood

198 of the resulting trees from TIPars was significantly higher than that of UShER (Fig. 3B).

Moreover, TIPars resulting trees tended to be very close to the reference tree with smaller log-

likelihood differences (fig. S2, A and B). A demonstration of the taxa-insertion was illustrated in

201 Fig. 2A by adding 1000 samples. We observed there were more crossing lines from reference tree

to UShER resulting tree indicating more misplaced insertions.

As to $16 \mathrm{~S}, \mathrm{H} 3 \mathrm{~N} 2$ and NDV datasets, TIPars mostly outperformed IQ-TREE2, PAGAN2 and

UShER with a significantly lower RF distance and a higher log-likelihood of resulting trees (Fig.

3, E to H; fig. S3). In the H3N2 dataset, there was no significant tree log-likelihood difference

between TIPars and UShER (Fig. 3G), and in NDV dataset, TIPars performed better than IQ-

TREE2 with higher mean log-likelihood but without statistical significance (Fig. 3H). The and PAGAN2 were less accurate than TIPars.

212 For the successive100 and successive1000 datasets, TIPars resulting trees had a significantly 
214 resulting trees was significantly higher than that of UShER (Fig. 3D; fig. S2, C and D). By

215 comparing the trees generated from TIPars and UShER (Fig. 2B), the difference is that TIPars

216 inserted some of query taxa (green lines in Fig. 2B; successive taxa pruned from the reference

217 tree) into two subtrees where one of them (the one containing over half the queries) had the same

218 topology as the one in the reference tree. Whereas UShER inserted those queries mostly within a

219 monophyletic clade but it was different from the reference tree. As a result, UShER retained the

220 local topology (better RF distance) (Lin et al., 2012; Smith, 2021) but missed the global topology

221 (worse log-likelihood). Through a RF distance comparison specifically to each query taxon

222 instead of all query taxa, we found that the RF distance resulted from UShER was not

223 significantly higher than that of TIPars (table S4).

225 On the other hand, we may suppose that in the situation of random100 and random 1000 tests, RF

226 distance would be a suitable metric for comparing the performance of taxa insertions as they are

227 similar to the case of single taxon placements, where most removed taxa are within different

228 monophyletic clades due to randomness (Bryant \& Steel, 2009).

230 To make the log-likelihood of the resulting trees comparable, we applied FastTree2 to reoptimize

231 the branch lengths with fixed topology (Price et al., 2010). However, compared to the efficiency

232 of taxa insertion (Table 1), the re-optimization is time-consuming. For example, the optimization

233 for a SARS2-100k tree took 10 to 12 hours and required around 125 GB memory (table S5).

234 Therefore, we also computed the log-likelihoods with fixed branch lengths (FLL) using IQ-

235 TREE2, and TIPars still outperformed UShER significantly (fig. S4) by achieving a higher log-

236 likelihood in the resulting tree output directly from the program. 
239 To verify practicability of TIPars in adding novel sequences into a given phylogeny, we further

240 performed an experiment to insert novel real-world SARS-CoV-2 samples into the SARS2-100k

241 reference tree. We randomly selected SARS-CoV-2 samples from GISAID which were not

242 included in the SARS2-100k dataset. Twenty sets of 100, 1000, 5000 and 10000 genome

243 sequences were generated as the queries for taxa insertion using TIPars and UShER.

245 Log-likelihoods of the resulting trees from each program were calculated and their pairwise 246 differences between TIPars and UShER were used to evaluate the performance. RF distance was 247 not a suitable metric in this experiment as a comparable reference tree was not available. TIPars 248 provided a resulting tree with a significantly better log-likelihood than UShER in all situations (p249 values <0.05; Fig. 4A).

251 In addition to tree log-likelihood, we also compared the PANGO lineages (PANGOlins) 252 assignment of the added samples (Rambaut et al., 2020) to validate the accuracy. Only 253 PANGOlins that existed in the reference tree were considered. We assigned each newly inserted 254 sequence with the lineage name of the subtree under the parental node of the inserted position. 255 The subtree was annotated by its descendant reference taxa if all of them were monophyletic 256 (McBroome et al., 2021). A true positive was defined as when the assigned lineage of a query 257 sequence was identical to its original PANGOlins label. In case of queries within unannotated 258 subtrees, we ignored them in the calculation. TIPars outperformed UShER by achieving higher true positive samples on the 100, 1000, 5000 and 10000 insertion datasets with an average of $92 \%$ 260 PANGOlins accuracy. The superiority of TIPars was statistically significant under a right-tailed 261 paired t-test (p-values < 0.001) on the 1000, 5000 and 10000 datasets (Fig. 4B and table S6).

\section{Discussion}


264 TIPars showed promising taxa placement and insertion accuracy in the phylogenies with

homogenous (H3N2 and SARS2-100k) and divergent (16S and NDV) sequences, and in

extremely large phylogeny (SARS2-660k) with reasonable runtime and memory usage. Although

UShER has a lower accuracy in the divergent sequence datasets (16S and NDV), it ran faster than

TIPars (Table 1).

Reconstruction of ancestral sequences are associated with all taxa across the phylogenetic tree,

which could be done using maximum likelihood statistical models or other advanced techniques

(Ishikawa et al., 2019; Kosakovsky Pond et al., 2020; Pupko, Pe'er, Shamir, \& Graur, 2000; Y.

Yu et al., 2020). So ancestral sequences may reveal more accurate (especially intermediate)

evolutionary information than the consensus mutation lists along each individual lineage as

UShER does. The evolutionary information can be used to distinguish insertion, deletion and

substitution events in the searching of taxon placement (Löytynoja \& Goldman, 2005), which

may help TIPars to be robust on more divergent phylogenies (Loytynoja et al., 2012). Overall,

compared to existing phylogenetic placement programs, TIPars is a robust method for a variety of

datasets with densely sampled and highly similar sequences of a single species which are

common in tracking pathogen epidemiology and transmission, as well as the sequences with

greater intraspecific divergence such as the genome datasets at genus, families or higher

taxonomic levels for systematics studies.

Although we showed that TIPars resulting trees with higher tree log-likelihood compared to other

programs, a general limitation of the phylogenetic placement method is that errors from incorrect placements accumulate as multiple sequences are inserted sequentially. In order to minimize the error due to large numbers of sequence insertions, it is suggested to conduct tree refinements on not only branch length but also tree topology using different techniques such as nearest-neighbor 
interchanges (NNIs) and subtree-pruning-regrafting (SPRs) (Price et al., 2010). Furthermore,

starting such optimization process with an initial tree of higher log-likelihood may achieve a final tree with better log-likelihood using certain of time (Price et al., 2010). As demonstrated in table S7, for the resulting trees of equal RF distance from both TIPars and UShER $(n=28)$, the branch length optimized trees for TIPars had higher $(n=14)$ or equal $(n=12)$ tree log-likelihoods than the ones resulted from UShER.

TIPars could facilitate the future development of sequence analysis methods that make use of the phylogenetic placement information. For instance, genome assembly of NGS read data from the metagenome can use phylogenetic positions of the short-read sequences to distinguish between related microbial strains or lineages. With the aid of TIPars, NGS sequences could be inserted to the branches of specific strains or lineages in a reference phylogeny. This can be used in calculating the proportion of strains in mixed infection even when one of the strains is at low abundance in which de novo assembly may generate incomplete contigs.

Since the start of the COVID-19 pandemic, over 5 million SARS-CoV-2 genome sequences have been made publicly available (Shu \& McCauley, 2017). With the reduction in cost, the rate of genome sequencing is expected to skyrocket in the future. By providing rapid and memory efficient taxa insertions at high accuracies, TIPars may improve real-time tracing and monitoring of SARS-CoV-2 transmission through the large-scale global phylogenetic analysis of the everincreasing SARS-CoV-2 genome sequences. 
314 After assigning the ancestral sequences at every internal node and taxa sequences at external

315 nodes, TIPars inserts a set of new samples into the reference phylogenetic tree sequentially based

316 on parsimony criteria.

318 For a query sequence Q, TIPars computes the minimal substitution score against every branch in

319 the tree. While inserting query Q into to the branch A-B (parent node - child node) at a potential

320 newly added node P (Fig. 1A), the minimal substitution score is the sum of substitution scores

321 that sequence Q differs from both sequence A and sequence B based on a specific substitution

322 scoring table based on the IUPAC nucleotide ambiguity codes (table S3). The single branch with

323 the minimum substitution score $\sigma$ is reported as the best placement.

However, in terms of multiple placements where more than one branch have the same minimum

substitution score, TIPars applies simple but practical rules to filter them to a single best

placement such that multiple queries would be inserted sequentially based on one resulting tree.

The first priority is to select the branch with node A containing the most numbers of child nodes.

The second priority is to select the branch with node A of the lowest node height, that is the total

branch length on the longest path from the node to a leaf (Suchard et al., 2018). Finally, in the

case where the ambiguity cannot be resolved by the first two priorities, TIPars just turns to a pick

up randomly. Even though TIPars will filter out multiple placements, these potential placements

will also be printed out for user notice.

335 We proposed a local estimation model to calculate the pendant length of the newly introduced branch P-Q $\left(l_{P-Q}\right)$ which is considering the branch lengths of the local triplet subtree $(\mathrm{A},(\mathrm{B}, \mathrm{Q}))$

(Fig. 1A). Pendant length is defined as $l_{P-Q}=\sigma /\left(\delta_{A}+\delta_{B}\right) * l_{A-B}$, where $\delta_{A}$ and $\delta_{B}$ are the unique 
location of $\mathrm{P}$ on branch A-B is determined by the ration of $\delta_{A}$ and $\delta_{B}$, i.e., Distal length:

$l_{A-P}=\delta_{A} /\left(\delta_{A}+\delta_{B}\right) * l_{A-B}$, and Sibling length: $l_{B-P}=\delta_{B} /\left(\delta_{A}+\delta_{B}\right) * l_{A-B}$. The ancestral sequence

341 of node $\mathrm{P}$ is estimated by majority vote of the nucleotide bases of sequence $\mathrm{A}, \mathrm{B}$ and $\mathrm{Q}$. To retain

342 the topology of reference tree, a potential nucleotide base of Q will be only derived from A or B.

343 For a special case of $l_{A-B}$ is zero but $\sigma$ is not, TIPars will consider upper branch of A's parent to

344 A for scaling.

We implemented TIPars using Java with BEAST library (Suchard et al., 2018). Both FASTA and

VCF formats are acceptable for loading sequences while NEWICK format is for the tree file.

FASTA file is the default setting, but VCF file is more memory efficient for large dataset of high

similar sequences, e.g. SARS-CoV-2 virus. To convert a FASTA file to VCF file with all

sequence mutations, i.e. insertion, deletion and substitution, we used a Python package

PoMo/FastaToVCF.py (Schrempf, Minh, De Maio, von Haeseler, \& Kosiol, 2016).

\section{Benchmark datasets preparation}

Unmasked SARS-CoV-2 MSA from GISAID was downloaded on 6th July 2021. Then all SARS-

CoV-2 viral genome sequences collected before 1st January 2021 were extracted from the MSA.

In order to ensure the sequences used for downstream analysis were complete, SARS-CoV-2

genomes with sequence length $<29,000 \mathrm{bp}$ and $>0.5 \%$ Ns were removed (namely

GISAID202101). To ensure that the global phylogenetic diversity is well represented in the subsampled dataset, sequences from all lineages as designated by the PANGO nomenclature system

(Rambaut et al., 2020) were sub-sampled. Where fewer than 50 sequences of a given lineage were 
364 19/Wuhan/WIV04/2019/EPI_ISL_402124 included as the reference genome (namely SARS2-

365 100k). The SARS2-100k reference tree was then built using IQ-TREE2 with GTR model using 366 the EPI_ISL_402124 as root. Ancestral sequences of each internal node were estimated using

367 PastML with the MSA and the IQ-TREE2 generated tree as input.

Three small but representative nucleotide sequence datasets namely, bacterial 16S rRNA (16S),

hemagglutinin genes of human seasonal influenza A viruses (H3N2), and Newcastle disease virus

genomes (NDV), were prepared for programs performance comparison. The $16 \mathrm{~S}$ dataset was

372 downloaded from Genomes OnLine Database (Mukherjee et al., 2019) and randomly down-

373 sampled to 800 sequences. HA sequences of $800 \mathrm{H} 3 \mathrm{~N} 2$ viruses were randomly extracted from

374 Influenza Research Database (Zhang et al., 2017). The 235 NDV sequences were downloaded

375 from GenBank. Alignments were constructed using MUSCLE (Edgar, 2004). Reference trees of

376 these datasets were built using RAxML (Stamatakis, 2014) standard hill-climbing heuristic search

377 with 100 multiple inferences and GTRGAMMA model. Ancestral sequences were estimated

378 using ML joint method (Pupko et al., 2000).

\section{Novel SARS-CoV-2 query sequence dataset}

To generate novel query sequences for the 20 sets of 100, 1000, 5000 and 10000 sequences,

SARS-CoV-2 genomes that were not included in the SARS2-100k dataset were randomly

384 selected from the GISAID202101 dataset. Selected sequences were then aligned to the SARS2-

385 100k sequences alignment by opening necessary gaps to obtain the full-length MSA. The newly

386 selected sequences were extracted to obtain the final query sample sets. Corresponding new gaps

387 were also added back to the ancestral sequence alignment for each dataset generated. PANGO 
388 lineages data for the novel SARS-CoV-2 query sequences and the taxa of reference tree was

389 downloaded from GISAID on 6th July 2021.

391 Benchmark programs

393 We compared TIPars to four state-of-the-art phylogenetic placement tools, namely UShER, EPA-

394 ng, IQ-TREE2 and PAGAN2 while EPA-ng only works for single taxon placement and IQ-

395 TREE2 and PAGAN2 were only used for multiple taxa insertion.

397 For the SARS2-100k dataset, only TIPars and UShER were considered as the other programs

398 were not able to complete the computation within a reasonable runtime (Table 1). For the three

399 smaller datasets, we compared all of them comprehensively. Details of the commands used for

400 different programs could be found in table S8.

402 TIPars, UShER and EPA-ng would report multiple placements for single taxon insertion. The 403 marked best placements of TIPars and UShER by themselves were used for our accurac

404 evaluation. EPA-ng reports its results sorted by log-likelihood, so the placement with the highest 405 log-likelihood was applied for assessment.

407 For any tools that accept only binary tree, i.e., EPA-ng and PAGAN2, we first converted the 408 original polytomous tree to a binary tree using the Ape R package (Paradis \& Schliep, 2019).

When adding unaligned query samples, it is suggested to align them to the MSA of taxa and 411 ancestral sequences in the reference tree using MAFFT ('--add' option) (Katoh \& Standley, 412 2013). 


\section{Evaluation metrics}

415 For single taxon placement evaluation, we first pruned one taxon from the reference tree and re-

416 inserted it back. To assess the consistency between placement algorithms and the typical tree-

417 constructing approach, we proposed using Robinson-Foulds (RF) Distance as a measure of the

418 tree topology accuracy, as calculated by TreeCmp (Bogdanowicz, Giaro, \& Wróbel, 2012). When

419 the RF distance between a hypothetical tree and the reference tree is zero, the topology of the

420 hypothetical tree is the same as the reference tree which means the algorithm inserts the query

421 sample into the reference tree topological correctly. Another performance comparison with

422 different true positive definition was conducted for binary trees derived from trees with polytomy

423 using the measurement of whether sister node sets are identical to reference (Y. Turakhia et al.,

424 2020).

426 For multiple taxa insertion evaluation, we randomly pruned a set of taxa from the reference tree

427 and re-inserted them back. In addition to using RF distance to compare the hypothetical tree

428 against the reference tree, we also calculated the log-likelihood of the hypothetical tree as a

429 measurement of the accuracy of the taxa insertions. We applied two methods to compute log-

430 likelihoods including FastTree2 (double-precision version) (Gamma20 Log-Likelihood) (Price et

431 al., 2010) for optimized branch length, and IQ-TREE2 (Log-Likelihood (Fixed Br)) for fixed

432 branch length.

434 EPA-ng outputs the placement information (placed branch, distal length, and pendant length) for a 435 query without the construction of the final tree. In order to compute the RF distance, we assisted 436 EPA-ng in inserting the query into the reference tree to generate the hypothetical tree. 
IQ-TREE2 and PAGAN2 support initial tree, but they are not exactly based on the input tree

topology for construction, so RF distance to original reference tree is not suitable for them.

440 Note that UShER outputs the final constructed tree using the number of mutations as branch

441 length (otherwise no branch length would be specified at branches modified), so we modified its

442 branch length as number of mutations divided by alignment length in calculation of log-likelihood 443 with fixed branch length model.

\section{Statistics}

$44799 \%$ t-test confident intervals and 99\% paired t-test p-value (right tail) for the results of TIPars 448 against other programs were computed by Matlab R2013b. All violin graphs were generated by R 449 4.1.1 using the package ggstatsplot (Patil, 2021). Illustration and annotation of phylogenetic trees 450 were done using the R package ggtree (G. Yu, Smith, Zhu, Guan, \& Lam, 2017).

\section{Data and materials availability}

454 SARS2-CoV-2 data used in this work were all downloaded from GISAID

455 (https://www.gisaid.org/). TIPars is available at https://github.com/id-bioinfo/TIPars.

\section{Acknowledgments}

459 We gratefully acknowledge the following Authors from the Originating laboratories responsible 460 for obtaining the specimens and the Submitting laboratories where genetic sequence data were 461 generated and shared via GISAID Initiative, on which this research is based. A full 462 acknowledgement table can be found with two EPI_SET-IDs, i.e., EPI_SET_20211201vz and 
463 EPI_SET_20211206tc, in Data Acknowledgement Locator under GISAID resources

464 (https://www.gisaid.org/).

This project is supported by the Hong Kong Research Grants Council's General Research Fund

(17150816), the National Natural Science Foundation of China's Excellent Young Scientists Fund

(Hong Kong and Macau) (31922087), the Health and Medical Research Fund (COVID1903011-

WP1), the Innovation and Technology Commission's InnoHK funding ( $\left.\mathrm{D}^{2} 4 \mathrm{H}\right)$, and the

Guangdong Government for the funding supports (2019B121205009, HZQB-KCZYZ-2021014,

200109155890863, 190830095586328 and 190824215544727).

\section{Competing interests}

Authors declare that they have no competing interests.

\section{References}

Barbera, P., Kozlov, A. M., Czech, L., Morel, B., Darriba, D., Flouri, T., \& Stamatakis, A. (2019). EPA-ng: Massively Parallel Evolutionary Placement of Genetic Sequences. Syst Biol, 68(2), 365-369. doi:10.1093/sysbio/syy054

Bogdanowicz, D., Giaro, K., \& Wróbel, B. (2012). TreeCmp: Comparison of Trees in Polynomial Time. Evolutionary Bioinformatics Online, 8, 475-487. doi:10.4137/EBO.S9657

Bryant, D., \& Steel, M. A. (2009). Computing the Distribution of a Tree Metric. IEEE ACM Trans. Comput. Biol. Bioinform., 6, 420-426.

Edgar, R. C. (2004). MUSCLE: a multiple sequence alignment method with reduced time and space complexity. BMC Bioinformatics, 5, 113. doi:10.1186/1471-2105-5-113

Filipski, A., Tamura, K., Billing-Ross, P., Murillo, O., \& Kumar, S. (2015). Phylogenetic placement of metagenomic reads using the minimum evolution principle. BMC Genomics, 16(1), S13. doi:10.1186/1471-2164-16-S1-S13

Ishikawa, S. A., Zhukova, A., Iwasaki, W., \& Gascuel, O. (2019). A Fast Likelihood Method to Reconstruct and Visualize Ancestral Scenarios. Molecular Biology and Evolution, 36(9), 2069-2085. doi:10.1093/molbev/msz131

Katoh, K., \& Standley, D. M. (2013). MAFFT Multiple Sequence Alignment Software Version 7: Improvements in Performance and Usability. Molecular Biology and Evolution, 30(4), 772-780. doi:10.1093/molbev/mst010 
Kosakovsky Pond, S. L., Poon, A. F. Y., Velazquez, R., Weaver, S., Hepler, N. L., Murrell, B., ... Muse, S. V. (2020). HyPhy 2.5-A Customizable Platform for Evolutionary Hypothesis Testing Using Phylogenies. Molecular Biology and Evolution, 37(1), 295-299. doi:10.1093/molbev/msz197

Lin, Y., Rajan, V., \& Moret, B. M. E. (2012). A Metric for Phylogenetic Trees Based on Matching. IEEE/ACM Transactions on Computational Biology and Bioinformatics, 9(4), 1014-1022. doi:10.1109/TCBB.2011.157

Löytynoja, A., \& Goldman, N. (2005). An algorithm for progressive multiple alignment of sequences with insertions. Proceedings of the National Academy of Sciences of the United States of America, 102(30), 10557. doi:10.1073/pnas.0409137102

Loytynoja, A., Vilella, A. J., \& Goldman, N. (2012). Accurate extension of multiple sequence alignments using a phylogeny-aware graph algorithm. Bioinformatics, 28(13), 1684-1691. doi:10.1093/bioinformatics/bts198

Matsen, F. A., Kodner, R. B., \& Armbrust, E. V. (2010). pplacer: linear time maximumlikelihood and Bayesian phylogenetic placement of sequences onto a fixed reference tree. BMC Bioinformatics, 11(1), 538. doi:10.1186/1471-2105-11-538

McBroome, J., Thornlow, B., Hinrichs, A. S., Kramer, A., De Maio, N., Goldman, N., . . . Turakhia, Y. (2021). A Daily-Updated Database and Tools for Comprehensive SARSCoV-2 Mutation-Annotated Trees. Molecular Biology and Evolution. doi:10.1093/molbev/msab264

Minh, B. Q., Schmidt, H. A., Chernomor, O., Schrempf, D., Woodhams, M. D., von Haeseler, A., \& Lanfear, R. (2020). IQ-TREE 2: New Models and Efficient Methods for Phylogenetic Inference in the Genomic Era. Mol Biol Evol, 37(5), 1530-1534. doi: 10.1093/molbev/msaa015

Moon, J., \& Eulenstein, O. (2019, 2019//). The Cluster Affinity Distance for Phylogenies. Paper presented at the Bioinformatics Research and Applications, Cham.

Mukherjee, S., Stamatis, D., Bertsch, J., Ovchinnikova, G., Katta, H. Y., Mojica, A., . . Reddy, T. (2019). Genomes OnLine database (GOLD) v.7: updates and new features. Nucleic Acids Res, 47(D1), D649-D659. doi:10.1093/nar/gky977

Paradis, E., \& Schliep, K. (2019). ape 5.0: an environment for modern phylogenetics and evolutionary analyses in R. Bioinformatics, 35(3), 526-528. doi:10.1093/bioinformatics/bty633

Patil, I. (2021). Visualizations with statistical details: The 'ggstatsplot' approach. PsyArXiv. doi:10.21105/joss.03167

Price, M. N., Dehal, P. S., \& Arkin, A. P. (2010). FastTree 2--approximately maximum-likelihood trees for large alignments. PLoS One, 5(3), e9490. doi:10.1371/journal.pone.0009490

Pupko, T., Pe'er, I., Shamir, R., \& Graur, D. (2000). A fast algorithm for joint reconstruction of ancestral amino acid sequences. Mol Biol Evol, 17(6), 890-896. doi:10.1093/oxfordjournals.molbev.a026369

Rambaut, A., Holmes, E. C., O’Toole, Á., Hill, V., McCrone, J. T., Ruis, C., . . Pybus, O. G. (2020). A dynamic nomenclature proposal for SARS-CoV-2 lineages to assist genomic epidemiology. Nature Microbiology, 5(11), 1403-1407. doi:10.1038/s41564-020-0770-5

Robinson, D. F., \& Foulds, L. R. (1981). Comparison of phylogenetic trees. Mathematical Biosciences, 53(1), 131-147. doi:https://doi.org/10.1016/0025-5564(81)90043-2

Schrempf, D., Minh, B. Q., De Maio, N., von Haeseler, A., \& Kosiol, C. (2016). Reversible polymorphism-aware phylogenetic models and their application to tree inference. $J$ Theor Biol, 407, 362-370. doi:10.1016/j.jtbi.2016.07.042

Shu, Y., \& McCauley, J. (2017). GISAID: Global initiative on sharing all influenza data - from vision to reality. Euro surveillance : bulletin Europeen sur les maladies transmissibles = 
European communicable disease bulletin, 22(13), 30494. doi:10.2807/15607917.ES.2017.22.13.30494

Smith, M. R. (2021). Information theoretic generalized Robinson-Foulds metrics for comparing phylogenetic trees. Bioinformatics, 37(14), 2077-2078. doi:10.1093/bioinformatics/btab200

Stamatakis, A. (2014). RAxML version 8: a tool for phylogenetic analysis and post-analysis of large phylogenies. Bioinformatics, 30(9), 1312-1313. doi:10.1093/bioinformatics/btu033

Suchard, M. A., Lemey, P., Baele, G., Ayres, D. L., Drummond, A. J., \& Rambaut, A. (2018). Bayesian phylogenetic and phylodynamic data integration using BEAST 1.10. Virus evolution, 4(1), vey016. doi:10.1093/ve/vey016. (Accession No. 29942656)

Turakhia, Y., Thornlow, B., Hinrichs, A. S., De Maio, N., Gozashti, L., Lanfear, R., . . CorbettDetig, R. (2020). Ultrafast Sample Placement on Existing Trees (UShER) Empowers Real-Time Phylogenetics for the SARS-CoV-2 Pandemic. bioRxiv. doi:10.1101/2020.09.26.314971

Turakhia, Y., Thornlow, B., Hinrichs, A. S., De Maio, N., Gozashti, L., Lanfear, R., . . CorbettDetig, R. (2021). Ultrafast Sample placement on Existing tRees (UShER) enables realtime phylogenetics for the SARS-CoV-2 pandemic. Nature Genetics, 53(6), 809-816. doi:10.1038/s41588-021-00862-7

Yu, G., Smith, D. K., Zhu, H., Guan, Y., \& Lam, T. T.-Y. (2017). ggtree: an r package for visualization and annotation of phylogenetic trees with their covariates and other associated data. Methods in Ecology and Evolution, 8(1), 28-36. doi:https://doi.org/10.1111/2041-210X.12628

Yu, Y., Blair, C., \& He, X. (2020). RASP 4: Ancestral State Reconstruction Tool for Multiple Genes and Characters. Molecular Biology and Evolution, 37(2), 604-606. doi:10.1093/molbev/msz257

Zhang, Y., Aevermann, B. D., Anderson, T. K., Burke, D. F., Dauphin, G., Gu, Z., . . . Scheuermann, R. H. (2017). Influenza Research Database: An integrated bioinformatics resource for influenza virus research. Nucleic Acids Res, 45(D1), D466-D474. doi:10.1093/nar/gkw857 
Figures and Tables

A

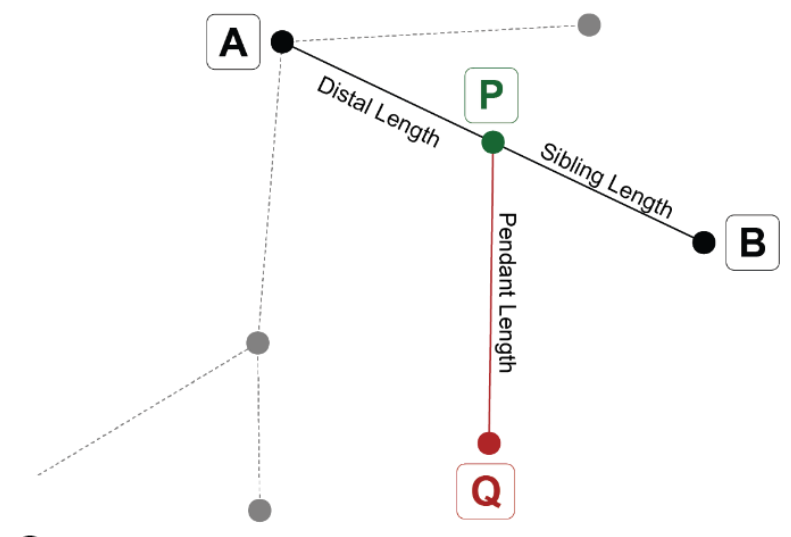

C

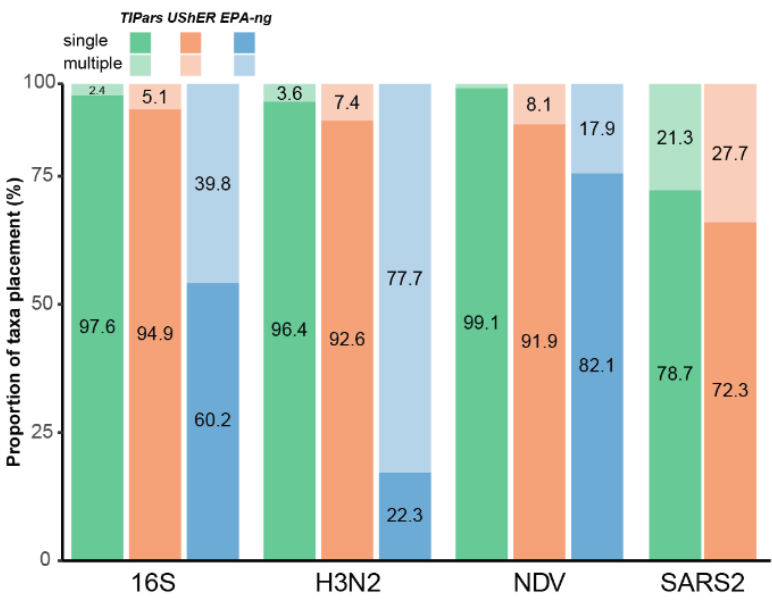

B

D
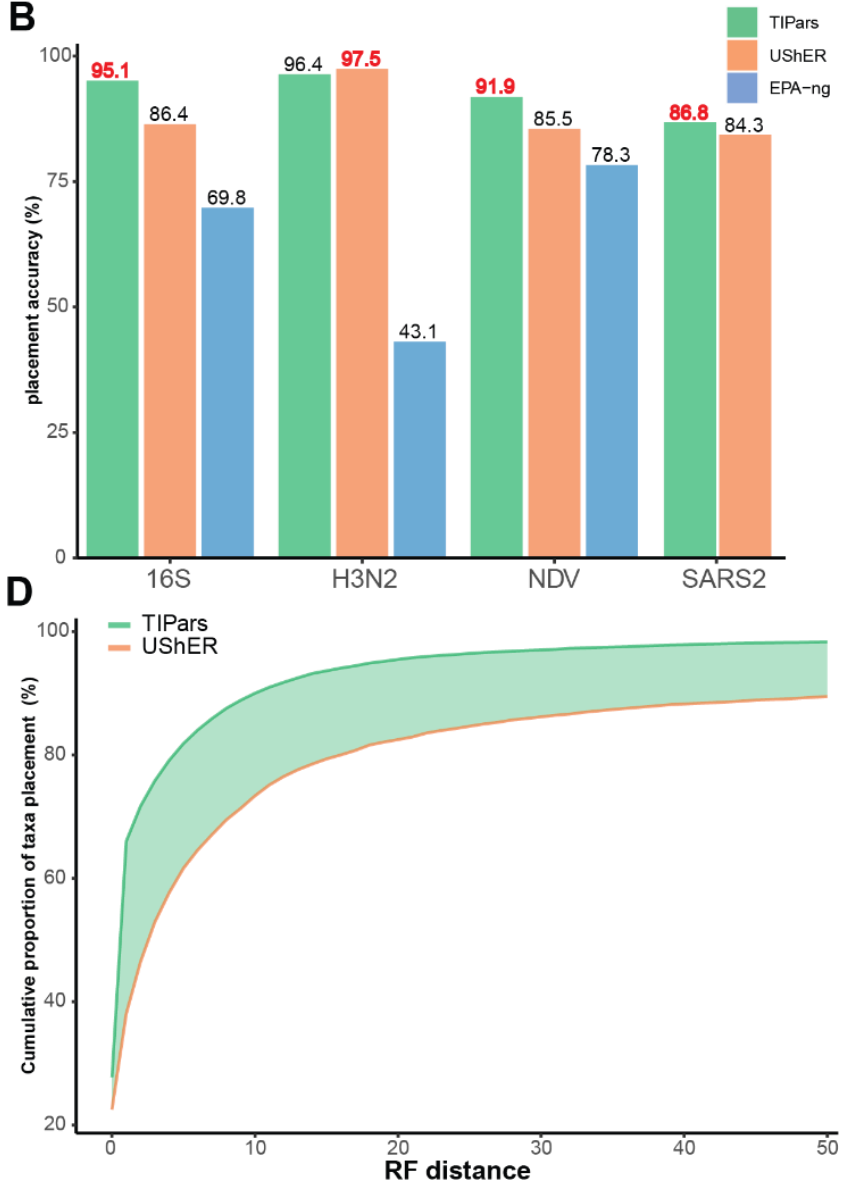

Fig. 1. Illustration of phylogenetic placement and single taxon placement performance. (A)

Illustration of the placement for a query sequence. "Q" indicates the query sequence, " $\mathrm{A}$ " and "B"

represent the existing nodes in the reference tree. "P" represents the parental node of "Q" generated by TIPars. Minimum substitution score is calculated based on the triplet formed by AB-Q. (B) Bar charts represent the accuracy of single taxon placement on 16S, H3N2, NDV and SARS2-100k datasets using TIPars, UShER and EPA-ng respectively. Accuracy is indicated on top of each bar and the highest accuracy in each dataset is highlighted in red. (C) Stacked bar charts show the proportion of single and multiple taxon placement result for TIPars (Green), UShER (Orange) and EPA-ng (Blue) on the 16S, H3N2, NDV and SARS2-100k datasets.

Proportion with $>1 \%$ is indicated within the bar. (D) Cumulative proportion of single taxa 
bioRxiv preprint doi: https://doi.org/10.1101/2021.12.30.474610; this version posted January 3, 2022. The copyright holder for this preprint (which was not certified by peer review) is the author/funder, who has granted bioRxiv a license to display the preprint in perpetuity. It is made available under aCC-BY-NC-ND 4.0 International license.

590 placement on the SARS2-660k dataset with different RF distance cutoff. Highlighted area

591 represents the difference between TIPars and UShER. 
A

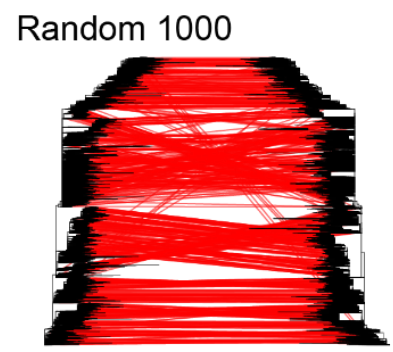

RF: 364.5

LL: -1954051

C

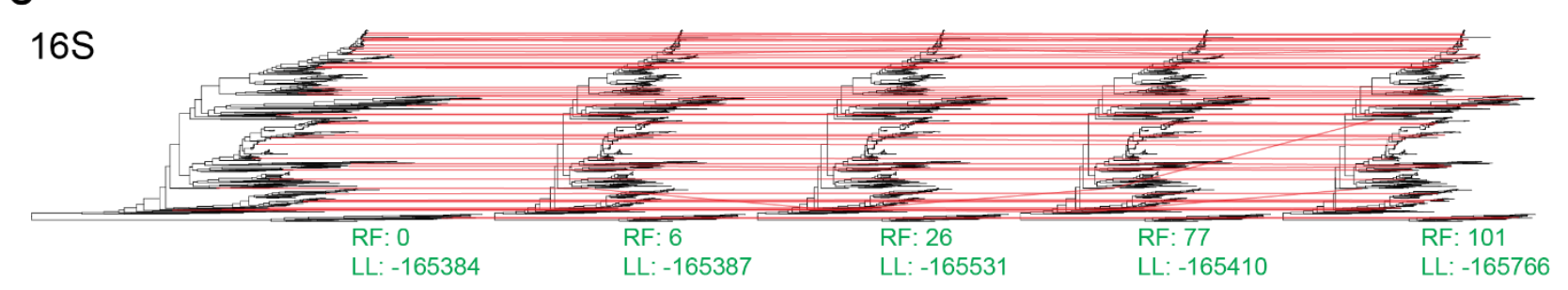

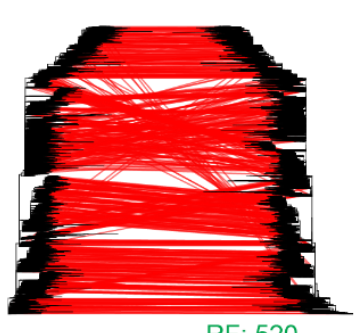

RF: 520

LL: -1954218
B

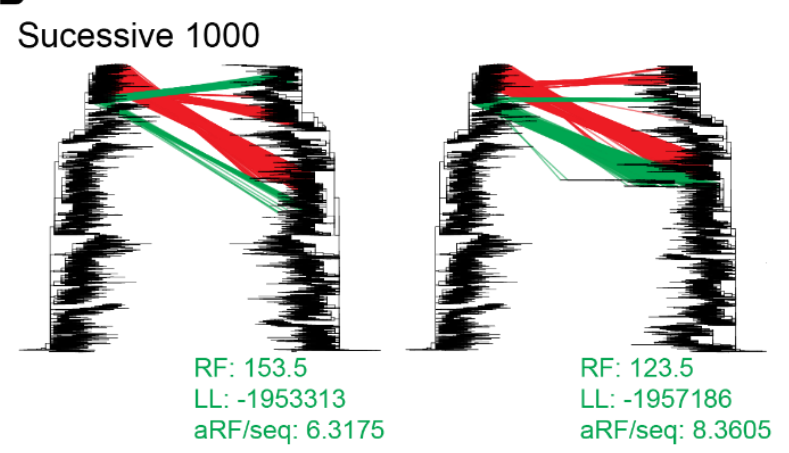

H3N2

Fig. 2. Taxa insertion visualization. (A) A demonstration of TIPars resulting tree (Left) and

UShER resulting tree (Right) paired with the reference SARS2-100k reference tree (Left tree in

both figures) for the insertion of randomly selected 1000 taxa sequences. Red lines link the

corresponding positions of inserted taxa between reference and resulting tree. (B) A

demonstration of TIPars resulting tree (Left) and UShER resulting tree (Right) paired with the

reference SARS2-100k reference tree (Left tree in both figures) for the insertion of successively

selected 1000 taxa sequences. Green lines indicate different taxa insertion positions between 
604 taxa in NDV, 16S (Midpoint rooted) and H3N2 datasets. From the left to the right are trees of

605 reference, TIPars, UShER, IQ-TREE2 and PAGAN2. RF distance (RF) compared to the reference

606 tree and the Gamma20 log-likelihood (LL) are shown at the bottom of each resulting tree. 
A

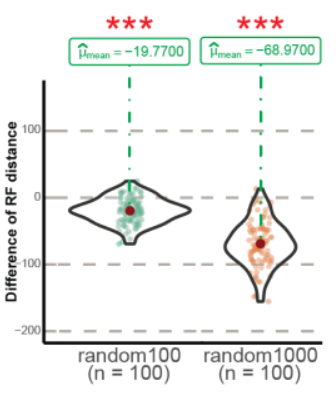

B
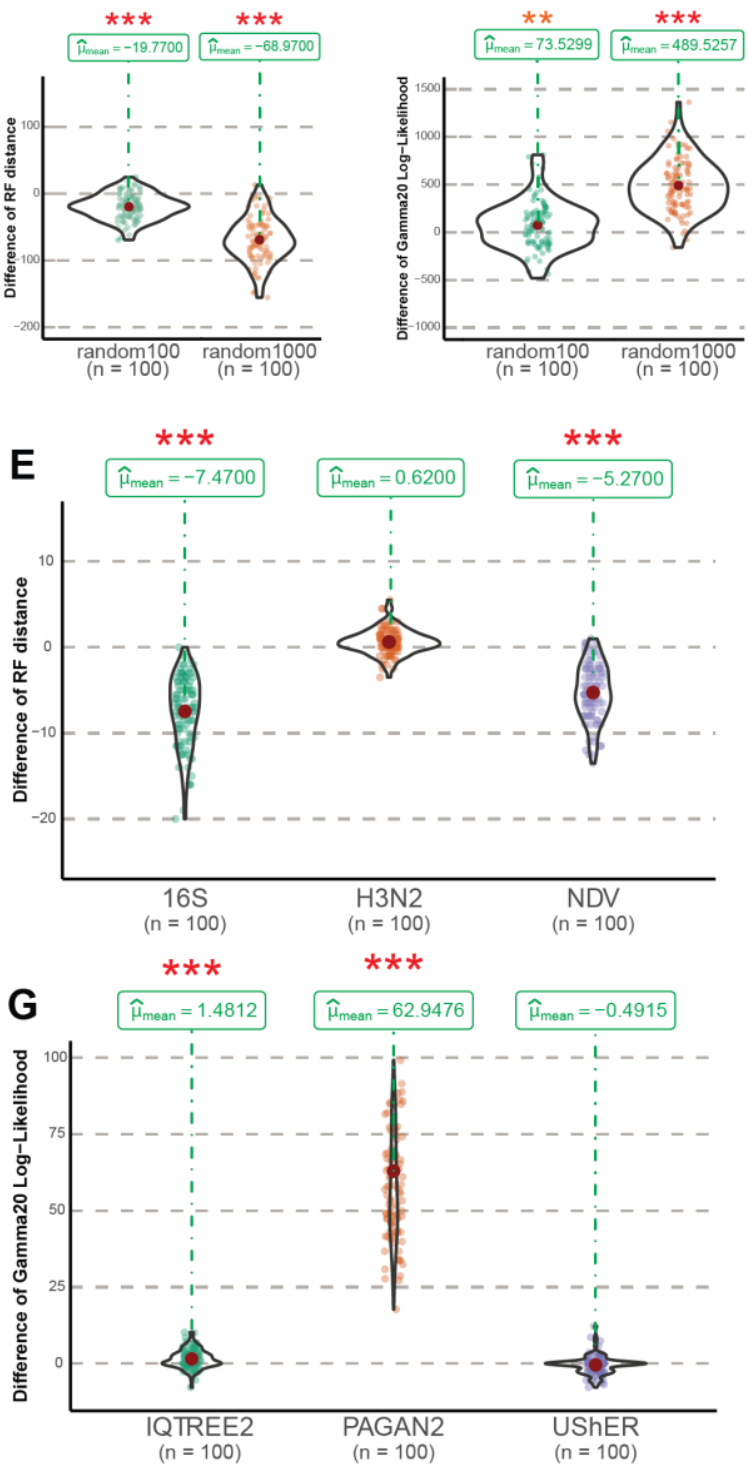

C
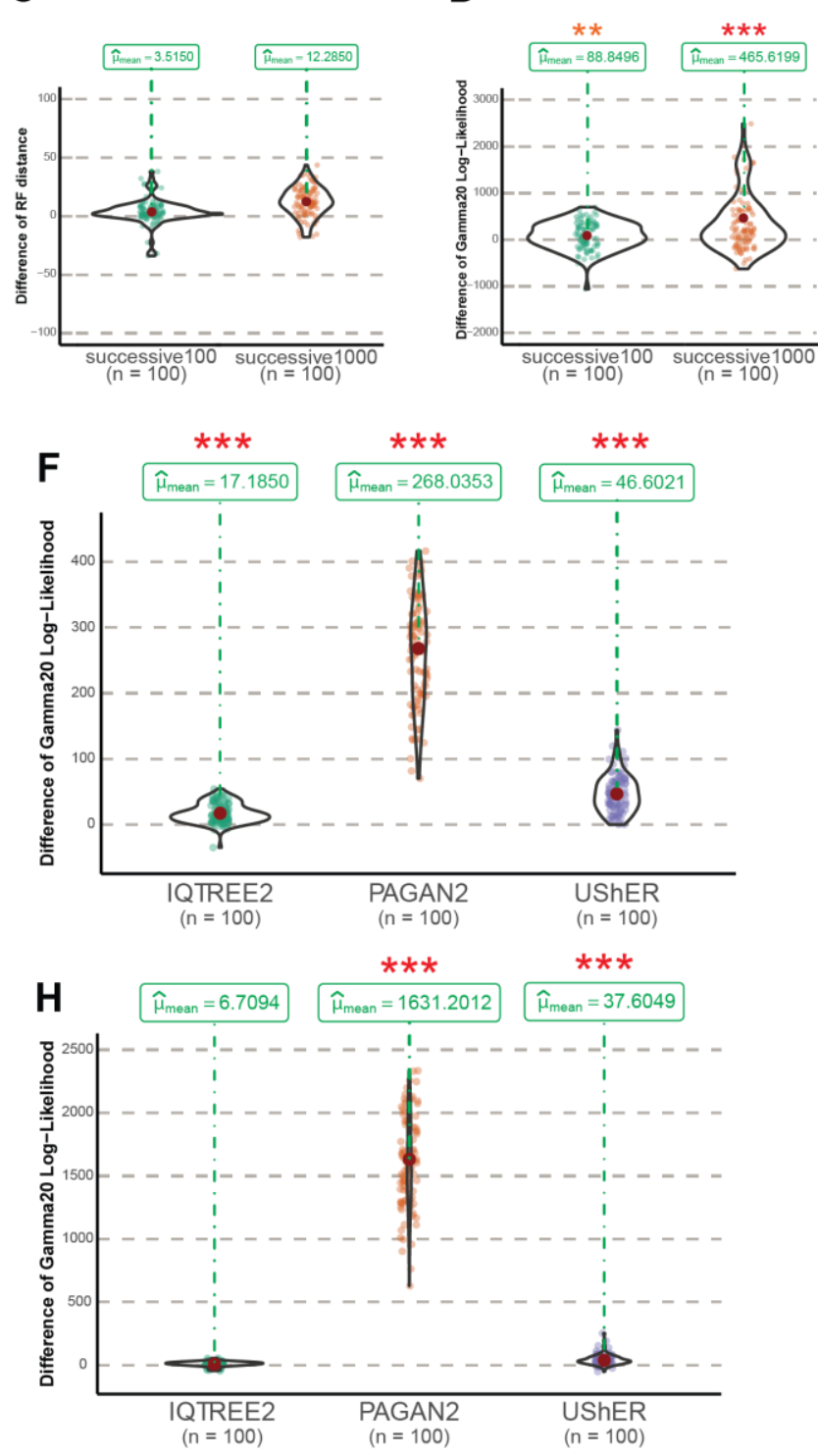

Fig. 3. Multiple sequences insertion performance. (A-D) Violin graphs show the distribution of paired differences of the RF distance and the Gamma20 log-likelihood between the optimized resulting trees generated by TIPars and UShER (TIPars - UShER) for the random 100, 1000 and

613 successive 100 and 1000 multiple sequences insertions. (E) Distribution of the paired difference

614 of the RF distance between the optimized resulting trees generated by TIPars and UShER (TIPars

615 - UShER) on 16S, H3N2 and NDV random 50 multiple sequences insertions. (F-H) Distribution of the paired difference of the Gamma20 log-likelihood between the optimized resulting trees 617 generated by TIPars and the three other programs (TIPars - Others) on 16S (F), H3N2 (G) and 
618 NDV $(\mathbf{H})$ random 50 multiple sequences insertions. P-value for the right-sided paired t-test is

619 indicated by the asterisk on top of each violin diagram, where $\mathrm{p}<0.05$ is indicated by one pink

620 asterisk $(*), \mathrm{p}<0.01$ by two orange asterisks $(* *)$ and $\mathrm{p}<0.001$ by three red asterisks $(* * *)$. 


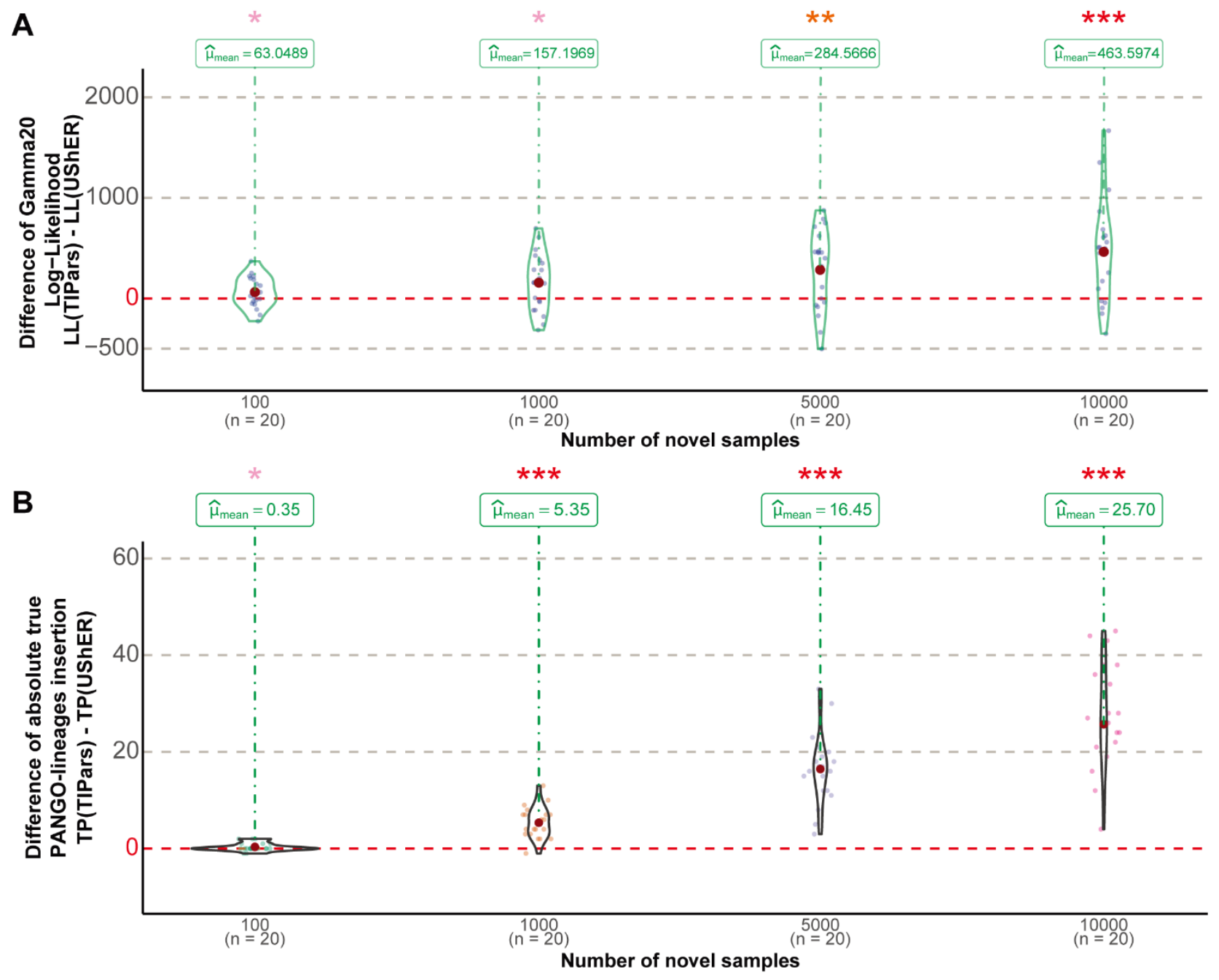

624 Fig. 4. Performance of inserting actual novel sequences. (A, B) Violin graph represents the 625 distribution of the paired differences between the Gamma20 log-likelihood (LL) (A) and the 626 absolute number of true PANGO-lineages insertion (TP) (B) of TIPars over UShER. p-value for 627 the right-sided paired t-test was indicated by the asterisk on top of each violin diagram, where $628 \mathrm{p}<0.05$ indicated by one pink asterisk $(*), \mathrm{p}<0.01$ by two orange asterisks $(* *)$ and $\mathrm{p}<0.001$ by 629 three red asterisks $(* * *)$. 


\section{Table 1. Average running time and memory used through 10 repeated runs of}

632 inserting/placing 100 genome samples into SARS2-100k reference tree. Tests were running on

633 a server of 64 Intel Xeon Gold 6242 CPU cores and 1500 GB RAM. We also compared TIPars

634 with UShER on a general computer with 8 CPU cores. TIPars ran with a JAVA setting of

635 Xmx1G. The running time of UShER contains its necessary computation of 'mutation-annotated

636 tree'. PAGAN2 was not runnable for this dataset. N/A indicates that data are not applicable.

\begin{tabular}{lllll}
\hline Tools & CPU cores & Mean insertion & Mean running & Mean peak \\
& assigned & time (HH:MM:SS) & time (HH:MM:SS) & memory (GB) \\
\hline TIPars & 64 & $0: 00: 21$ & $0: 00: 52$ & 1.39 \\
\hline TIPars & 8 & $0: 00: 31$ & $0: 01: 03$ & 1.18 \\
\hline UShER & 64 & $0: 00: 02$ & $0: 03: 14$ & 0.84 \\
\hline UShER & 8 & & & 0.16 \\
\hline EPA-ng & 64 & $0: 00: 05$ & $0: 05: 14$ & 1022.14 \\
\hline IQ-TREE2 & 64 & N/A & $0: 10: 25$ & 101.10 \\
\hline PAGAN2 & 64 & N/A & N:45:A & \\
\hline
\end{tabular}

